\title{
Toxocara Brain Infection in Pigs is Not Associated with Visible Lesions on Brain Magnetic Resonance Imaging
}

\author{
Alessandra Nicoletti, ${ }^{1}$ Luis A. Gomez-Puerta, ${ }^{2,3 *}$ Gianfranco Arroyo, ${ }^{3}$ Javier Bustos, ${ }^{3,4}$ Armando E. Gonzalez, ${ }^{2,3}$ and \\ Hector H. Garcia ${ }^{3,4}$ for The Cysticercosis Working Group in Peru \\ ${ }^{1}$ Dipartimento G.F. Ingrassia, Università di Catania, Catania, Italia; ${ }^{2}$ School of Veterinary Medicine, Universidad Nacional Mayor de San Marcos, \\ Lima, Peru; ${ }^{3}$ Center for Global Health, Universidad Peruana Cayetano Heredia, Lima, Peru; ${ }^{4}$ Cysticercosis Unit, Instituto Nacional de Ciencias \\ Neurologicas, Lima, Peru
}

\begin{abstract}
Human exposure to Toxocara spp. is very frequent, and its larvae can cross the blood-brain barrier and invade the central nervous system (CNS), causing neurotoxocariasis. We aimed to establish a neurotoxocariasis animal model in pigs confirmed by necropsy. Also, the presence of larvae in the CNS was assessed using magnetic resonance imagings (MRIs), to establish brain lesions caused by the larvae migration. Ten pigs were infected intraperitoneally with 3,000 Toxocara larvae. Cerebral toxocariasis was evaluated using MRls at days 7, 14, 21, and 49, and pigs were euthanized after the examination. Brain tissues were examined by microscopy, and five pigs presented Toxocara, most frequently at day 21 after infection. None of the 10 pigs showed alterations on MRls. Our study confirms that intraperitoneal Toxocara infection produces neurotoxocariasis in pigs. Toxocara larvae passage through the brain does not seem to produce lesions detectable at MRls.
\end{abstract}

Human toxocariasis is a zoonosis caused by the larval stages of Toxocara canis and Toxocara cati, the common roundworms of dogs and cats. Humans are infected by accidental ingestion of embryonated Toxocara eggs present in contaminated soil or food, or by the ingestion of encapsulated larvae contained in the raw tissues of paratenic hosts, such as cattle and chickens. ${ }^{1}$ When ingested, embryonated eggs develop into juvenile larvae that crossing the small intestine migrate to any organ through the circulatory system determining a multisystemic inflammatory tissue reaction. Visceral and ocular larva migrans are the most common clinical manifestations. Toxocara larvae can cross the blood-brain barrier, invading the central nervous system (CNS) and leading to neurotoxocariasis. ${ }^{2}$

Despite consistent evidence that exposure to Toxocara is very common, CNS infection in humans is thought to be rare, and to date, only about 100 cases of neurotoxocariasis have been reported in the literature since $1956 .^{2-4}$ Clinical presentations of neurotoxocariasis include a wide spectrum of neurological manifestations including seizures, meningitis, encephalitis, myelitis, and cerebral vasculitis. ${ }^{5,6}$ Neurotoxocariasis is rarely considered in the differential diagnosis of neurological pathologies, and the disease is probably underdiagnosed. ${ }^{2}$

Animal models serve as a comparison to human toxocariasis, although migration routes and predilection sites depend on the host species. Toxocara larvae generally migrate to the brain in animal models including rodents, pigs, primates, and others. $^{7}$ Among animal models, pigs have been largely adopted because of physiological and biochemical similarities, particularly in terms of immune response. ${ }^{7}$ In pigs, Toxocara larvae exhibit an extensive migration pattern and induce a strong immune response, ${ }^{8}$ with a lack of clinical symptoms despite extended larval migration and the presence of pathological changes in different neural tissues. ${ }^{9}$ Toxocara larvae

*Address correspondence to Luis A. Gomez-Puerta, Department of Veterinary Epidemiology and Economics, School of Veterinary Medicine, Universidad Nacional Mayor de San Marcos, Av. Circunvalacion 2800, San Borja, Lima 41, Peru. E-mails: lucho92@yahoo.com or Igomezp@unmsm.edu.pe can be recovered from the lymph nodes around the intestines, from the lungs, liver, and brain of experimentally infected pigs on days 10-21 postinfection, but totally disappeared after 126 days postinfection. ${ }^{8-10}$

Pathology in porcine cerebral toxocariasis demonstrates congestion, edema, shrinkage of nerve cells, vacuolization, gliosis, satellitosis, neurophagia, and liquefactive necrosis. ${ }^{11}$ Disease in the pig is self-limited, with a significant decrease in larval numbers over time and a tendency of lesions to heal from 28 days postinfection onward. ${ }^{10}$

This study aimed to establish an animal model using intraperitoneal inoculation of $T$. canis larvae in pigs confirmed by necropsy and microscopical diagnosis. In addition, the presence of larvae in the CNS was also assessed using magnetic resonance imagings (MRIs), to establish if the passage of larvae through the brain may produce lesions detectable by neuroimaging.

Ten pigs of approximately 8 weeks of age, of both sexes and from a technified farm, were used. The piglets were kept in the animal facilities of the School of Veterinary Medicine from the National University of San Marcos, Lima, Peru, and fed on commercial food and sterile water ad libitum.

Toxocara eggs were collected directly from the uterus of adult nematodes obtained from naturally infected dogs (treated with pyrantel pamoate). The eggs were placed in a $2.5 \%$ potassium dichromate solution for 30 days until the larvae developed. Subsequently, egg hatching was induced for larvae to come out. Larvae viability was determined by observation of movement under a microscope.

Infectivity of the larvae was evaluated in 20 BALB/c mice, in which 500 larvae were inoculated intraperitoneally (IP). Five days postinfection, mice were euthanized with $60 \mathrm{mg} / \mathrm{kg}$ sodium pentobarbital (IP) and the presence of Toxocara larvae in liver and lung tissues was evaluated using pepsin digestion. Tissues were cut with scissors into small pieces and digested for 24 hours at $37^{\circ} \mathrm{C}$ in a solution consisting of $1 \%$ pepsin and $1 \% \mathrm{HCl}$ in tap water, and sedimented in $50-\mathrm{mL}$ conic tubes by centrifugation. The sediment was examined under microscopy at $\times 40$ of magnification to detect larvae. ${ }^{12}$ After confirming viable larval infection in mice, the pigs were infected IP 


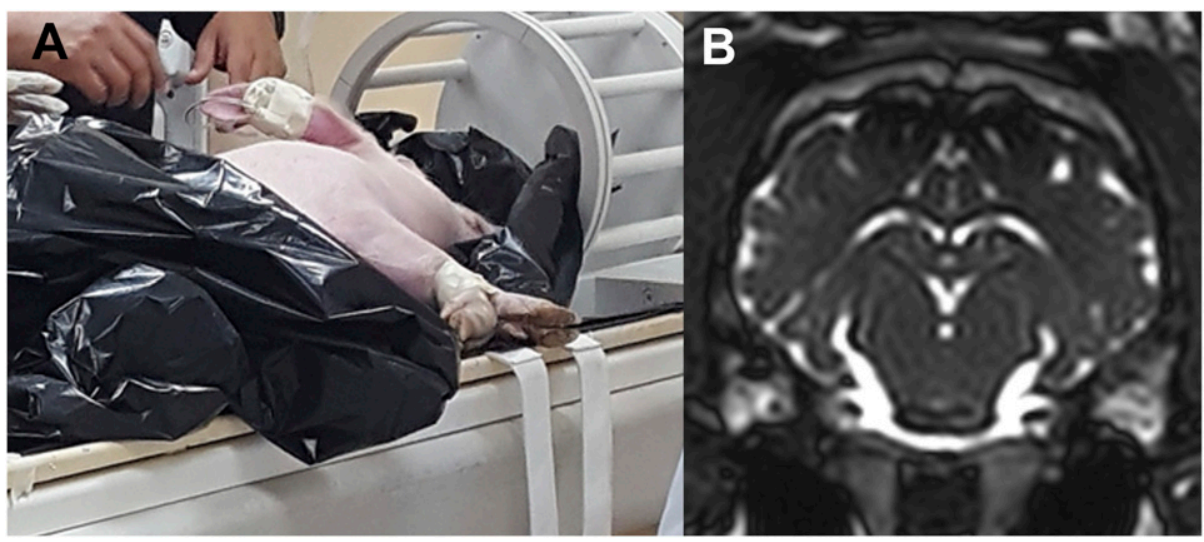

FIGURE 1. Cerebral magnetic resonance imaging procedure (A) and image (B) on a participant pig. This figure appears in color at www.ajtmh.org.

with 3,000 larvae, under anesthesia with intramuscular ketamine at $20 \mathrm{mg} / \mathrm{kg}$ and xylacine at $1.5 \mathrm{mg} / \mathrm{kg}$.

Cerebral toxocariasis in pigs was evaluated using MRls at day 7 (two pigs), day 14 (two pigs), day 21 (three pigs), and day 49 (three pigs). Before MRls, pigs were anesthetized with ketamine xylacine as described earlier, and then, a catheter was inserted into the marginal ear artery of each pig and kept latent by very slow normal saline drip with ketamine as needed (Figure 1). Magnetic resonance imagings were performed on a 3-Tesla MRI scanner (Philips Achieva, Best, the Netherlands). Sequences included coronal, sagittal, and axial turbo field echo T1-weighted gradient echo images under conditions of repetition time $=7 \mathrm{~ms}$, echo time $=4 \mathrm{~ms}$, flip angle $=8^{\circ}$, pixel bandwidth $=270 \mathrm{kHz}$, section thickness $=0.5-4 \mathrm{~mm}$, and matrix $=256-480$ pixels, before and after injection of a contrast solution (gadolinium diethylene triaminopentaacetic acid at $0.1 \mathrm{mmol} / \mathrm{kg}$ ) through the ear IV catheter.

After MRls, pigs were euthanized with a lethal intravenous dose of $120 \mathrm{mg} / \mathrm{kg}$ of sodium pentobarbital. The pigs' brains were retrieved at necropsy. All brain tissue was used for the detection of Toxocara larvae. The brain was divided into left and right hemispheres, as well as anterior and posterior regions. Also, the cerebellum was totally evaluated. Small pieces of the brain and cerebellum tissue of approximately $5 \mathrm{~mm}^{3}$ were crushed between two glass slides, and the presence of Toxocara larvae was diagnosed under microscopy at $\times 40$ of magnification.

The study was approved by the Institutional Ethics Committees for the Use of Animals at the Universidad Peruana Cayetano Heredia (protocol number 032-09-18). Treatment of animals adhered to the Council for International Organizations of Medical Sciences International Guiding Principles for Biomedical Research Involving Animals.

All pigs underwent brain MRls with contrast injection at different times between day 7 and day 49 after infection as described earlier. None of the 10 pigs showed alterations on MRIs.

Necropsy and MRls were performed in the same day. Toxocara larvae were found in the brain tissues of five of the 10 study pigs: $0 / 2$ at day $7,1 / 2$ at day 14 (three larvae in the right hemisphere, two in the anterior region and one in the posterior region), all three pigs at day 21 (pig 1: one larva in the posterior region of the right hemisphere; pig 2: three larvae in the left hemisphere, two in the anterior region and one in the posterior region; and pig 3: five larvae in the right hemisphere, three in the anterior region and two in the posterior region; one larva in the posterior region of left hemisphere; and three larvae in the cerebellum), and $1 / 3$ at day 49 (one larva in the right posterior region) (Figure 2).

Our study confirms that intraperitoneal infection of Toxocara larvae produces neurotoxocariasis in pigs. In particular, in agreement with the literature, all pigs sacrificed 21 days postinfection presented Toxocara larvae in the brain. Our findings also support the hypothesis that larvae do not seem to persist for prolonged periods; literature, in fact, suggests that larvae totally disappeared after 126 days postinfection, indicating self-limiting factors. ${ }^{10}$

We assessed the presence of Toxocara larvae in the brain using a 3-Tesla MRI. However, all encephalic MRls were negative, including the five pigs in which the presence of the larvae in the brain was demonstrated by necropsy. Reported cases of human neurotoxocariasis are supported by neuroimaging findings and involve diverse clinical presentations. ${ }^{4}$ Beyond reported clinical cases and case series, several epidemiological studies have suggested a possible association between Toxocara seropositivity neurological disorders, without neuroimaging confirmation. ${ }^{2}$ In particular, several case-control studies and two meta-analyses suggest a possible role of toxocariasis in the incidence of epilepsy. ${ }^{13}$

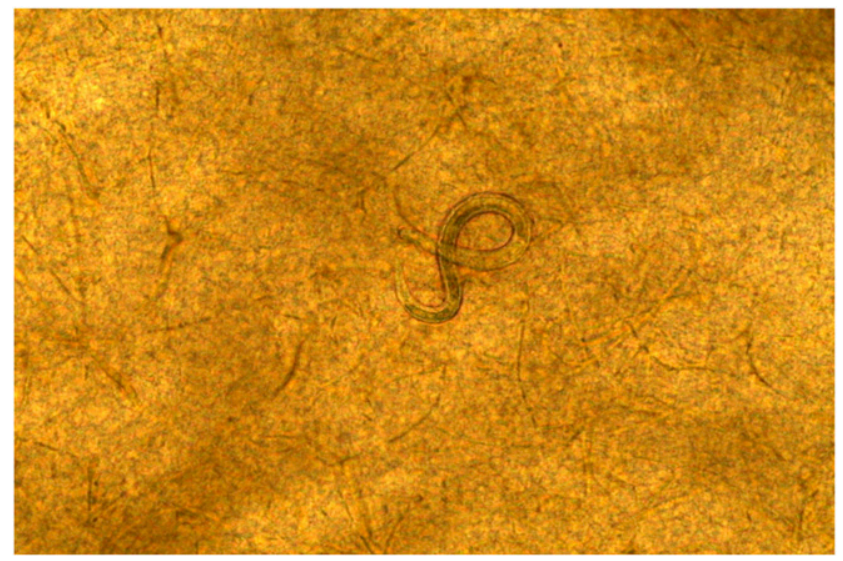

Figure 2. Toxocara canis larvae in the brain tissue. This figure appears in color at www.ajtmh.org. 
In animal models, larvae become undetectable in the brain after a certain period of time. ${ }^{10}$ The passage of Toxocara larvae through the brain does not seem to produce lesions detectable at MRls, suggesting that it does not necessarily produce structural lesions and is likely followed by complete resolution of lesions.

Diverse, nonspecific MRI lesions have been found in biopsy-confirmed cases of human neurotoxocariasis, including ring-enhancing lesions typical of a granulomatous reaction. ${ }^{4}$ Histological examination generally shows a granulomatous inflammatory response containing large numbers of eosinophils and neutrophils in the parenchyma with perivascular lymphocytic infiltrates, but larvae are rarely detected inside the lesion. ${ }^{3}$ An intuitive hypothetical explanation for the lack of MRI lesions in pigs with brain toxocariasis would be that the migration of the larvae across the brain not necessary lead to an inflammatory granuloma. The development of a granulomatous reaction depends on several factors, including the host immune response as well as the parasitic load. It should be noted that the parasitic load in our experiment is lower than that reported in other studies probably because of the lower number of larvae $(3,000)$ inoculated IP. The short time to MRIs could also contribute to explain our negative findings. In experimental infections in mice, granuloma formation was observed at 11 weeks postinfection. ${ }^{14}$

How larval Toxocara infection can result in seizures and epilepsy is yet unknown. Although seizures can be caused by a granulomatous reaction induced by Toxocara larvae, there are only a few proven cases in the literature. ${ }^{4,15}$ In experimental studies in animals, cerebral Toxocara infection produces a marked immune response that disrupts the permeability of the blood-brain barrier, increasing proinflammatory cytokines and leading to neuronal damage. ${ }^{2}$ Toxocariasis also leads to autoantibody production, ${ }^{16}$ and autoantibodies against neuronal elements have been involved in some type of human epilepsies. ${ }^{17-20}$ In this scenario, the lack of abnormal MRI findings during the passage of Toxocara larvae suggests that epileptogenic mechanisms are unlikely to be due to a focal, granulomatous reaction. Further studies in the pig model can contribute to determine whether the other proposed mechanisms (blood-brain barrier disruption, inflammation, neuronal damage, and autoantibody production) contribute to seizures in neurotoxocariasis and define the underlying pathophysiological mechanisms.

Received December 7, 2019. Accepted for publication April 10, 2020.

Published online May 18, 2020.

Financial support: This research was funded by the Department of Medical and Surgical Sciences and Advanced Technologies "G.F. Ingrassia," the University of Catania, Italy (Piano Triennale di Sviluppo delle Attività di Ricerca Scientifica del Dipartimento 2016-2018) and the Fogarty International Center at the National Institutes of Health (FIC-NIH training Grant TW001140).

Disclaimer: Funders had no role in the study design, experimental phase, analysis, interpretation, writing the manuscript, or the decision to submit the manuscript.

Authors' addresses: Alessandra Nicoletti, Dipartimento G.F. Ingrassia, Università di Catania, Catania, Italia, E-mail: anicolet@unict.it. Luis A. Gomez-Puerta and Armando E. Gonzalez, School of Veterinary Medicine, Universidad Nacional Mayor de San Marcos, Lima, Peru, E-mails: Igomezp@unmsm.edu.pe and agonzalezz@unmsm.edu.pe. Gianfranco Arroyo, School of Public Health and Management,
Universidad Peruana Cayetano Heredia, Lima, Peru, E-mail: arroyogianfranco@gmail.com. Javier Bustos and Hector H. Garcia, Center for Global Health, Universidad Peruana Cayetano Heredia, Lima, Peru, E-mails: javier.bustos@jhu.edu and hgarcia1@jhu.edu.

\section{REFERENCES}

1. Rubinsky-Elefant G, Hirata CE, Yamamoto JH, Ferreira MU, 2010. Human toxocariasis: diagnosis, worldwide seroprevalences and clinical expression of the systemic and ocular forms. Ann Trop Med Parasitol 104: 3-23.

2. Fan CK, Holland CV, Loxton K, Barghouth U, 2015. Cerebral toxocariasis: silent progression to neurodegenerative disorders? Clin Microbiol Rev 28: 663-686.

3. Deshayes S, Bonhomme J, de La Blanchardiere A, 2016. Neurotoxocariasis: a systematic literature review. Infection 44: 565-574.

4. Sanchez SS, Garcia HH, Nicoletti A, 2018. Clinical and magnetic resonance imaging findings of neurotoxocariasis. Front Neurol 9: 53.

5. Ma G, Holland CV, Wang T, Hofmann A, Fan CK, Maizels RM, Hotez PJ, Gasser RB, 2018. Human toxocariasis. Lancet Infect Dis 18: e14-e24.

6. Nicoletti A, 2013. Toxocariasis. Handb Clin Neurol 114: 217-228.

7. Strube C, Heuer L, Janecek E, 2013. Toxocara spp. infections in paratenic hosts. Vet Parasitol 193: 375-389.

8. Helwigh AB, Lind P, Nansen P, 1999. Visceral larva migrans: migratory pattern of Toxocara canis in pigs. Int J Parasitol 29: 559-565.

9. Taira K, Saeed I, Lind P, Murrell KD, Kapel CM, 2003. Population dynamics of Toxocara canis in pigs receiving a single or multiple infection. Parasitology 127: 593-602.

10. Sommerfelt IE, Rosa A, Duchene A, Degregorio O, Lopez C, Pisanu A, De Torres R, 2004. Toxocara canis in experimentally infected pigs: migratory pattern and tissue lesions. Vet Parasitol 125: 323-334

11. Sasmal NK, Acharya S, Laha R, 2008. Larval migration of Toxocara canis in piglets and transfer of larvae from infected porcine tissue to mice. J Helminthol 82: 245-249.

12. Alba-Hurtado F, Munoz-Guzman MA, Valdivia-Anda G, Tortora JL, Ortega-Pierres MG, 2009. Toxocara canis: larval migration dynamics, detection of antibody reactivity to larval excretorysecretory antigens and clinical findings during experimental infection of gerbils (Meriones unguiculatus). Exp Parasitol 122: $1-5$.

13. Luna J, Cicero CE, Rateau G, Quattrocchi G, Marin B, Bruno E, Dalmay F, Druet-Cabanac M, Nicoletti A, Preux PM, 2018. Updated evidence of the association between toxocariasis and epilepsy: systematic review and meta-analysis. PLoS Negl Trop Dis 12: e0006665.

14. Kayes SG, Oaks JA. 1978. Development of the granulomatous response in murine toxocariasis. Initial events. Am J Pathol 93: 277-294.

15. Levite M, Ganor Y, 2008. Autoantibodies to glutamate receptors can damage the brain in epilepsy, systemic lupus erythematosus and encephalitis. Expert Rev Neurother 8: 11411160.

16. Wagner RG, Newton CR, 2009. Do helminths cause epilepsy? Parasite Immunol 31: 697-705.

17. Obwaller A, Duchene M, Walochnik J, Wiedermann G, Auer H, Aspock $\mathrm{H}, 2004$. Association of autoantibodies against small nuclear ribonucleoproteins (snRNPs) with symptomatic Toxocara canis infestation. Parasite Immunol 26: 327-333.

18. Ganor Y, Goldberg-Stern H, Lerman-Sagie T, Teichberg VI, Levite $M, 2005$. Autoimmune epilepsy: distinct subpopulations of epilepsy patients harbor serum autoantibodies to either glutamate/AMPA receptor GluR3, glutamate/NMDA receptor subunit NR2A or double-stranded DNA. Epilepsy Res 65: $11-22$.

19. Levite M, 2002. Autoimmune epilepsy. Nat Immunol 3: 500.

20. Palace J, Lang B, 2000. Epilepsy: an autoimmune disease? $\checkmark$ Neurol Neurosurg Psychiatry 69: 711-714. 